\title{
Contribution of Artificial Intelligence and Virtual Worlds towards development of Super Intelligent AI Agents
}

\author{
Simran Koul
}

\begin{abstract}
Artificial Intelligence (AI) has always been a goal as well as a challenge in the scientific field with a whole new world being accessible to people by accomplishing various achievements in the past decades. However, in the last many years, tremendous popularity has been received by the interactive computer games involving multi-user virtual environment which has been the prime attraction for millions of users around the world. This paper focuses on AI agents along with exploring the progress towards super intelligent AI and the roles played by artificial intelligence and virtual worlds in its development. It follows a unique approach to give an unbiased view on the various problems and the relation between them. There are namely two benefits from this research: firstly, evolution in science and technology requires a profound understanding on the advanced AI agents in the virtual worlds, and secondly, the virtual worlds have always been a magnificent platform for research on the challenging problems faced in the domain of Artificial Intelligence.
\end{abstract}

Keywords: Artificial intelligence, autonomous intelligent agents, interactive computer games, machine learning, super intelligent AI, virtual worlds, MUVEs.

The idea of having a simulated world where people are able to interact virtually has always been the key attraction since many decades. Earlier, the term virtual reality was defined as a human-computer interface that would include stimulations and interactions in real-time by the use of multiple sensors which aims to provide appropriate display of human senses like vision, hearing, touch, smell, and taste. The expectations and demands from the virtual reality concept were far more than head-mounted displays, 3-D sound, sensory gloves, etc. which the technological capabilities found hard to satisfy during that time. Improvement of PC designs in blend with web innovation vigorously influenced advancement of one distinctive kind of virtual cooperation PC games. Massively multiplayer online pretending games (MMORPGs) turned out to be particularly broad. One of the most illustrative models is WoW (WorldofWarcraft)- with a large number of open records, and all the more critically a huge number of dynamic

Revised Manuscript Received on May 15, 2020.

* Correspondence Author

Simran Koul*, School of Computer Science and Engineering, Vellore Institute of Technology, Vellore, Tamil Nadu, India.Email: simran.koul@yahoo.com

(c) The Authors. Published by Blue Eyes Intelligence Engineering and Sciences Publication (BEIESP). This is an open access article under the CC BY-NC-ND license (http://creativecommons.org/licenses/by-nc-nd/4.0/)

\section{INTRODUCTION}

endorsers too. A few examinations have demonstrated that populace of players have a place with a wide age go, with segment attributes that broadly differ. Prevalence of this kind of virtual communication can likewise be perceived in the way that understudies go through as long as 20 hours seven days playing different online PC games. Populace size and assorted variety of clients engaged with these gaming universes speak to a truly important potential for various research examines. It is accounted for that driving game creating companies (such as Blizzard and EA Games) gathered and investigated enormous informational collections thinking about the player's conduct. Different organizations additionally perceived the capability of gaming - e.g., IBM examined the way fruitful playing of WoW prompts the improvement of key reasoning strategies and authority capacities. Further advancement of 3D PC games at one point prompted something all the more socially unpredictable - the improvement of MUVEs (multi-client virtual conditions). These virtual situations or "virtual universes" as they are frequently called, pulled in enormous consideration (for example - There, Open Wonderland, Active Worlds, Second Life, and so on.). A huge number of clients spoke to by means of symbols (energized human-like characters), found in them a spot to all the while associate and mingle. It ought to be seen that a few analysts utilize a term 3D virtual universes, as a more extensive classification when alluding to both-intuitive PC games and MUVEs. In spite of the fact that they are fundamentally the same as, it ought not to be overlooked that PC games and MUVEs have various goals. Be that as it may, remembering that virtual universes can be defined as a PC based recreated conditions, in which clients can associate between themselves or with artificial operators, this sort of phrasing is fairly justified and will be followed further in the paper. PC games and MUVEs will likewise be at the same time utilized as terms, contingent upon what ought to be brought up in a specific sentence.

Prominence and nonstop improvement of recently referenced virtual intelligent universes subsequently empowered for new research bearings to be opened in different scientific fields, including the field of Artificial Intelligence. Among other, these virtual universes are perceived as a productive ground for explore in independent shrewd operators, which will be in the focal point of this paper. Other than the field of AI itself, development of insightful operators can be identified as an exceptionally beneficial for virtual universes too. Consequently,

Published By:

Blue Eyes Intelligence Engineering \& Sciences Publication

(C) Copyright: All rights reserved. 


\section{Contribution of Artificial Intelligence and Virtual Worlds towards development of Super Intelligent AI Agents}

this point will be fundamentally examined from alternate points of view, viable on the one side, and progressively unique on the other-with the craving to expand and incorporate significant experiences originating from both, scholastic network and monetarily arranged industry.

The paper is composed in the accompanying request. In Section 2, the job and significance of AI in virtual universes will be examined. Section 3 will give an examination of number of AI methods expecting to give an insightful conduct of specialists. In Section 4 virtual specialists will be put in a more extensive hypothetical structure, planning to give a one of a kind way to deal with the subject of potential ramifications and necessities driving toward human-level AI operators. Finishing up comments will be expounded in Section 5. Toward the end, rich wellspring of deliberately picked references utilized for this exploration study will be recorded.

\section{The Contribution of AI in Virtual Worlds}

It ought to be seen that regardless of incomplete cover, virtual universes and prior referenced computer generated simulation speak to totally different ideas. One of the significant contrasts is reflected in the way that MUVEs and present day PC games share a typical property that vary them from old augmented simulation thoughts - the majority of the client sensation originates from the illustrations showed on the PC screen. Propelled 3D designs can be identified as the fundamental element of the gigantic achievement of virtual universes previously. Be that as it may, in spite of the way that best in class 3D illustrations acts extremely influential, it is faulty on the off chance that it can completely give two components that are identified as a key issue - inundation and collaboration. One should see that these two components are commonly trustworthy. As it was defined, submersion speaks to an emotional impression that client partakes in a reasonable encounter. So as to accomplish more significant level of inundation, graphical representation is fundamental yet not sufficient necessity. In this way, it isn't extraordinary to peruse that the job of designs in these virtual universes got to the meaningful part where it can no longer speak to a vital improvement of client's understanding. Also that in serious game industry, elevated level of designs sometime in the past turned out to be quite anticipated. Thus, venturing up to the following degree of credible and reasonable experience suggests that exploration endeavors must be progressively situated toward the conduct of the game occupants, as opposed to on the visual appearance of nature. It is even detailed that with progressively complex visual appearance of the recreated world, the need for increasingly complex gaming agents (non-player characters) is expanding. Artificial insight is perceived as a key instrument which can to a great extent add to virtual universes, since AI can make gaming agent's behavior all the more engaging and common. More significant level of life-like conduct absolutely influences client's drenching in an enormous degree. Accordingly, it isn't astonishing that the nature of the executed $\mathrm{AI}$ is perceived as one of the principle assessment standards of the fruitful games, with number of devoted books managing the common sense issues identified with it (e.g., Steve Rabin's "AI Game Programming Wisdom" series).

In the beginning of the field, scope of AI procedures utilized in games was exceptionally restricted, concentrating generally on a straightforward AI. Reasons were different: from the way that some AI procedures are amazingly entangled and require a lot of computational force, to the basic truth that at some point propelled AI in games is considered as superfluous. Also the illustrations utilized a large portion of the CPU power, in that way leaving limited quantity of handling assets for AI. This pattern was changed and all things considered throughout the years anyway a portion of the issues remained. Various endeavors were made previously, so as to diminish a solid hole between scholarly $\mathrm{AI}$ and game industry designers, since these two are frequently troubled with various natures of their objectives. All things considered, notwithstanding the various methodologies, PC gaming universes presumably speak to the "biggest business utilization of artificial intelligence". Great potential that lies in applying of academic AI research to virtual worlds is not beneficial just for their future turn of events, yet additionally for the field of AI itself. As it was watched, virtual universes with its rich substance speak to a difficult stage for cutting edge AI look into, particularly in the space of keen specialists.

\section{A. Game Agents}

Watching the previous, one could see that the conduct of game specialists, or gaming agents as they are normally alluded to, was among the principle focal points of game AI. Despite the fact that there are a few minor departure from what precisely qualifies as the gaming agents, broadly acknowledged definition is that gaming agents are for the most part virtual world characters that are not constrained by a human client (regardless of on the off chance that they are going about as adversaries, associates, or impartially situated supporting characters). As it was accounted for by certain creators, an undeniable differentiation considering the business game $\mathrm{AI}$ on the one side and scholarly AI on the other could be taken note. Gaming agents are might be the best pointer of contrasts between the two, thinking about the idea of their objectives. The motivation behind AI in games is somewhat straightforward - to make a superior, increasingly reasonable gaming experience for clients. This doesn't essential incorporate creation of a propelled AI framework. In number of situations gaming agents are not intended to really be canny, but instead to give a fantasy of keen conduct. One could consider it as a kind of "smart" cheating. From the designer's perspective, this methodology is somewhat legitimate and even empowered, in light of the fact that in enormous per cent straightforward hallucination of insight can have a similar impact as a progressively mind boggling AI. Also that by and large, it is less practical and calculation is likewise less difficult. Purported "acceptance of difficult ideas mistrust", has its foundations in the broadly known "Eliza impact". Despite the fact that the focal point of this paper isn't on the dream of knowledge, a few perspectives must be talked about so as to give a more profound comprehension of the subject by and large.

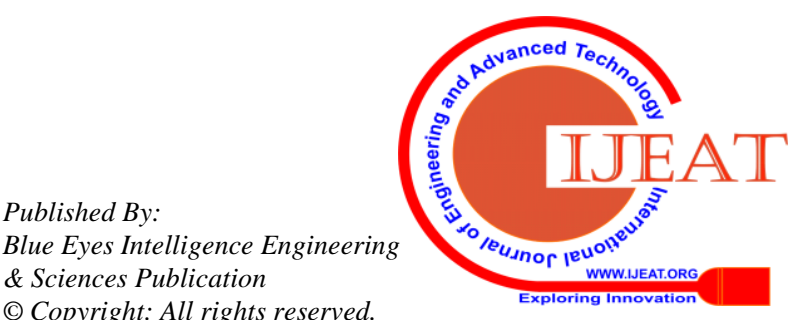


One of the illustrative models that are depicting this marvel can be found in a wide range of games where human client has PC controlled adversaries. In a typical situation, gathering of antagonistic operators is acting is some condition and they are conversing with each other: "Watch for your back", "Set up a perimeter", etc. Obviously, they are haphazardly shouting these expressions while simultaneously act totally free with no smart coordinated effort.

In any case, if this correspondence between gaming agents is painstakingly planned, it can regularly deliver a feeling of clever conduct for a human client taking an interest in a game, in that way expanding his degree of drenching into the virtual gaming condition. Other part of "cheating" is omniscience of the gaming agents. It is particularly observable when PC controlled adversary is playing against a human player in some game situation - in greater part of cases foe operator have ridiculous abilities, particularly with regards to looking, or dynamic speed. Identified with the past, one more viewpoint to be considered is the game difficulty. On the off chance that the gaming agent is practically top notch, at that point most of players will lose their advantage quick. Same thing will occur if gaming agents are too simple to even consider beating. So as to forestall this sort of situations engineers are attempting to accomplish an equalization by planning rivals that are not dumb, and yet not very shrewd. As it were, parts of tricking must be painstakingly actualized, so as to give a deception of wise conduct. Despite the fact that this sort of approach is propelled by gaming needs, a simple model can be found in scholarly AI. Alan Turing, one of the establishing fathers of AI, portrayed a kind of knowledge test (later notable as Turing test) and in his original work he broke down the circumstance where machines are not committing any errors: "It is claimed that the interrogator could distinguish the machine from the man simply by setting them a number of problems in arithmetic. The machine would be unmasked because of its deadly accuracy." In request to forestall this, straight forward arrangement is proposed machines should commit purposeful errors so as to mislead human investigative specialist.

\section{TECHNIQUES OF AI THAT CONTRIBUTE IN BEHAVIOR SHAPING OF GAMING AGENTS}

As it was at that point referenced, gaming agent's conduct is usually formed with a portion of the AI calculations. It is seen that scholastic AI and game AI have various perspectives on what qualifies as artificial knowledge. While game industry for the most part watches gaming agent's AI from a wide perspective, including even a few issues that have distinctive nature, scholastic network is frequently alluding to gaming agent's AI in a progressively thin sense, focusing just on the keen conduct. In this area uncommon accentuation will be on calculations and strategies utilized for dynamic and learning, as they speak to basic themes in regards to the underlining thought of the paper. I will briefly depict picked strategies, give models (business and scholarly) of their execution in regards to the subject of clever specialists, and examine a portion of the benefits and downsides accompanying their usage.

\section{A. Traditional Method Approaches}

Number of effective AI programs was created with Rule-based Systems, so it isn't amazing that they are utilized for control of gaming agent's decision at the very beginnings of the game AI. Their structure comprises out of accessible (information) and a lot of rules (in the event that rationale). Appropriately utilized, rule-based frameworks can give a modestly high level of control and sufficient power. Anyway they are once in a while utilized as a predominant strategy, since in greater part of cases there are less difficult and more efficient strategies to accomplish wanted decision.

Finite State Machines speak to a notable computational model. Despite the fact that not generally the most ideal arrangement, it is likely the most broadly utilized strategy in a game AI improvement. Number of fruitful PC games, for example, Half-Life arrangement or Quake arrangement utilized FSMs as a reason for the control of gaming agents. The thought is fairly straightforward: whenever, just one of a finite number of states is conceivable, and relying upon the sources of info that state could be changed. FSM is defined by its underlying state, rundown of potential states, and progress conditions. FSMs utilize a Boolean rationale; along these lines a state can be dynamic or latent (valid or bogus) Exchanging between the states, changes the behavior of the gaming agent. FSMs are anything but difficult to actualize, efficient (particularly with regards to straightforward gaming agent's behavior), smaller, and incredible calculation, which makes them somewhat great in a game turn of events. In any case, they are regularly scrutinized for being excessively inflexible, making them carry on wrong in perplexing and unpredicted situations. Further on, one of the primary hindrances in this methodology is that number of states can quickly congest, in the event that we misrepresent in multifaceted nature of the ideal behavior. This could be mostly evaded by bringing of sub-states into the frameworks, in that way making a various leveled finite state machines (HFSMs).

A few varieties of FSMs are conceivable, including Fuzzy State Machines where fluffy rationale is utilized as an option for the Boolean rationale. As a result, in contrast to the FSMs, framework could be in more than each state in turn. To be progressively exact, various degrees of enrolment can be appointed to states. Presentation of numerous states, just as fluffy rationale by and large, gives a feeling of increasingly characteristic and sensible gaming agent's behavior. Albeit sensibly easy to execute, it must be considered that such a large number of fluffy states could prompt a quick development of the framework intricacy known as "combinatorial blast". It ought to likewise be noticed, that other than the way that FuSMs have numerous focal points contrasted with standard FSMs (e.g., gaming agent's behavior is less unsurprising), the strategy is more vulnerable in the provisions of the difficult speculation. There are various instances of FuSMs and fluffy rationale in virtual universes announced in writing, particularly with regards to key and strategic games (e.g., Civilization: Call to Power).

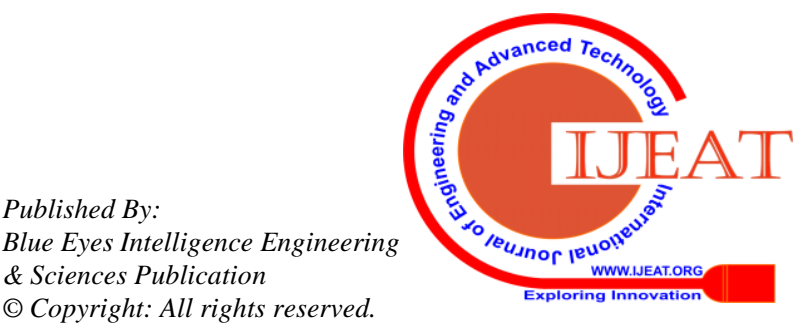




\section{Contribution of Artificial Intelligence and Virtual Worlds towards development of Super Intelligent AI Agents}

Other than state machines, scripting is utilized in greater part of virtual universes, with regards to building an AI framework. It ought to be noticed that contents are viewed as static and frequently will in general be mind boggling, which suggests their concern with consistency and difficulty scaling. This solo online AI procedure, which depends on reinforcement learning, expects to adjust AI to changing conditions on the web, while the game is being played. Calculation is effectively tried on the Neverwinter Nights business game.

Decision Trees are among the least complex dynamic components utilized in game AI. In short terms, this various leveled tree-like structure is composed in branch hubs and leaf hubs, where leaf hubs speak to potential choices. As it is depicted in, it executes separate and-vanquish system. Decision trees can be utilized alone, or in mix with other dynamic procedures. This calculation is sensibly quick, simple to alter, and easy to comprehend, as it was referenced toward the beginning. Other than dynamic, Decision tree learning is one of the most widely recognized methods for inductive derivation. One of the fundamental focal points lies in the way that this strategy is strong thinking about the missing information. Number of Decision tree learning calculations. Fascinating model, considering PC games, is the Black and White. Animals in this game use AI programming engineering called Belief-Desire-Intention, got from the hypothesis of human useful thinking. It depends on a few learning strategies, for example, generally known ID3 decision tree learning calculation, just as neural systems and reinforcement learning. Contingent upon climate the animal accomplishes something incorrectly or right, player can slap it (punishment, negative improvement) or stroke it (positive upgrade). Creatures recall player's criticism and afterward as per it adjust their decision.

Behavior Trees picked up their ubiquity in game AI people group, with Halo 2. In the next years, BTs became predominant strategy in game AI with number of various usages. In spite of the fact that not generally the most efficient (traversal issue), this strategy speaks to an integral asset for accomplishing of complex gaming agent's decision and significant level of control. In certain manner, BTs blend a few of leaving AI procedures and their qualities. BTs are working in a particular way, having undertakings/practices rather than states that are utilized in state machines. In spite of the fact that having a few similitudes with the prior referenced HFSMs, approach is somewhat imaginative. BTs comprehend huge numbers of the downsides found with state machines, such as upkeep issues. Evacuating or including of the specific state involves changes in the states of different states identified with it. Contingent upon number of states that are influenced, this can be somewhat hazardous as it progressively opens opportunities for mistakes. With BTs opportunities for mistakes is decreased, as hubs are carrying on autonomously and in this way are not influenced by changes in different pieces of the framework. Simple to keep up, reusable, versatile, extensible, and adaptable, it isn't amazing that decision trees became positive instrument for controlling of gaming agent's decision.

Engineers regularly utilize rather imaginative

methodologies so as to give life-like decision of the agents. The Sims is viewed as one of the games that vigorously influenced the field of game AI. In this life recreation PC game, player can provide arranges and watch the life of number of self-sufficient gaming agents, called Sims, while they connect with the earth. Sims "turned the idea of an AI back to front", with its "Smart Object" approach. Uniqueness of this idea lies in the way that enormous part of insight, particularly with respect to the dynamic, isn't fused in the gaming agents. In particular, gaming agents are furnished with the necessities, yet all the data considering the connection with some item are in the article itself.

\section{B. More Advanced Approaches due to Academic Research}

In complex virtual universes, gaming agents are confronted with a large number of potential communications. This makes actualizing of cutting edge AI very difficult. Along these lines, it isn't amazing that virtual universes for the most part depend on recently portrayed standard methodologies, which are very much demonstrated and altogether tested. For quite a while, further developed AI techniques that were essential considered as scholarly, were stayed away from. These calculations were frequently exceptionally entangled, computationally costly, and dangerous for execution, when contrasted with state machines. Prior in the content, we irregularly referenced learning on a few events. Gaming agents which can learn and adjust, speak to one of the interesting points to scholarly network, since the capacity to learn is one of the primary qualities of savvy conduct. Be that as it may, actualizing of learning calculations (particularly progressively) to gaming agents is as yet not generally applied in business PC games, and speaks to a risky undertaking for engineers. The principle reason lies in capriciousness. Elevated level of self-rule and unusual conduct that regularly accompanies propelled AI and AI is considered as unfortunate in games, as it can ruin the playability. From all the recently stated, it is clear why best in class AI calculations were not considered as the best fit for the on-going obliged frameworks, for example, intelligent PC games. All things considered, objective of the game architects was to make AI just as intricate as it was required, so their hesitance toward the more entangled and frequently nondeterministic approaches was to be expected. Further on, software engineers frequently didn't have the foggiest idea how to execute scholastic AI methods in a common sense way. Be that as it may, neural systems, developmental calculations, and other further developed techniques, step by step discovered their place in game AI. Now, one should see that usage of cutting edge AI doesn't infer that standard calculations, for example, state machines are superfluous. As it was seen, propelled AI frameworks frequently need to utilize a portion of these standard calculations at various framework levels. One of the illustrative models with regards to utilizing of scholastic techniques in business games is the F.E.A.R., a blockbuster FPS

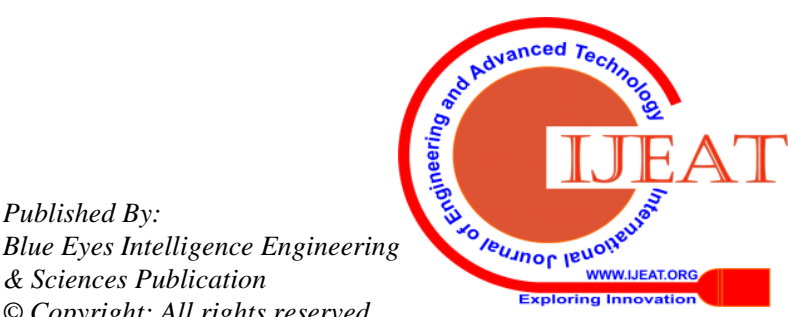


(first individual shooter) game. Among other, the game AI framework abuses the STRIPS (STanford Research Institute Problem Solver) rationale a spearheading mechanized organizer grew practically 50 years prior at Stanford University. It is additionally critical to make reference to SOAR subjective design, which was utilized for broad scholastic research on AI-based virtual characters. The Soar Quakebot, gaming agents tried in QuakeII game, expected to give increasingly responsive and flexible behavior. Dynamic of SOAR based insightful operators, was established in a see think-act cycle.

Further, expectation and expectation capacities were created, since expectation is perceived as one of the key highlights of clever conduct. It is accounted for that Soar Quakebot effectively tested even human rivals with middle of the road level playing limits.

It could be noticed that Bayesian hypothesis speaks to a foundation of the present AI. In this way it isn't astounding that its application to game AI stood out of scholastics. One of the early papers in the field explored Bayesian programming for learning of gaming agent practices in the Unreal Tournament. Engineers dealt with computational costs, which is significant issue for potential business applications. A few investigations were done thinking about the StarCraft. This constant system PC game, distributed by Blizzard Entertainment, increased huge prominence all around the globe. Bayesian model is created so as to anticipate the initial methodologies in the game. Further on, same creators presented a Bayesian probabilistic model for empowering gaming agents to settle on strategic dynamic and anticipate adversary's assaults.

Reinforcement Learning speaks to one of the significant AI zones of research [5]. As it was depicted, RL intends to empower specialist to learn by cooperating with the world, without solid oversight and without the specific model of the world [6]. Utilizing of RL in game AI, is accounted for to be fairly restricted, despite the fact that this interesting hypothesis offers preferences which are exceptionally significant for the field (e.g., adapting to erratic situations), depicts utilizing of RL idea so as to create group of gaming agents for playing the Unreal Tournament in control situation. Creators utilized modified Q-learning so as to empower gaming agents to upgrade dynamic systems. With regards to FPS games, broad research was additionally done reasoning that RL can be effectively executed in game AI. This paper additionally thought about progressive, rule-based, and flat RL control. Experimentation with MRL executed in Second Life virtual world gave important outcomes regarding the matter. Remembering, that clients of such virtual universes can change the earth by including or evacuating objects, gaming agents which can learn and adjust speak to an examination subject of high premium.

Neural Networks are more than effectively executed in table games, for example, Backgammon, and even profoundly complex Go [7]. With regards to intuitive PC games, utilizing of neural systems in game AI was frequently considered as entangled and computationally costly, and in this way for quite a while it was not all that normal. Anyway various effective instances of appropriate NN executions during the years indicated all the benefits originating from this strategy. One of the first and most significant instances of neural systems in games was found in the late nineties. Animals, a PC game perceived as one of the forward leaps in artificial life science, utilized neural systems for tangible engine coordination and behavior determination of engineered operators [8]. Firmly influenced by creature science - natural chemistry and hereditary calculation standards were additionally utilized for re-enactments. Neural systems are additionally actualized in a few business dashing games, for example, Forza Series or Colin McRae Rally. Forza Series hustling game distributed by Microsoft Studios created AI framework dependent on neural systems, called Drivatar. By investigation of gathered information and Bayesian learning, Drivatars are attempting to imitate genuine client's driving method. The more some client plays the game more information about this gaming conduct is accessible, in this way empowering the Drivatar to have a bigger level of comparability with the client. The point is to mimic specific highlights of person's driving style (how you brake, or use gas, and so forth.), in that way making AI operators that contrast one from another. The NERO (Neuro advancing Robotic Operatives) game, created at University of Texas, speaks to an intriguing case of non-business AI based game. Human player has a job of an educator to a group of operators (recreated robots). The objective is to set them up for a battle, while specialists start the preparation without any aptitudes, simply the capacity to learn. So as to empower specialists to learn, NERO use rtNEAT (constant Neuro advancement of Augmenting Topologies) calculation for developing progressively complex neural systems continuously. Not at all like the scripting where inevitably shortcomings can be distinguished and abused, this methodology is focusing on gaming agents to adjust and improve their behavior by learning. Neuro evolution, a mix of hereditary calculations and neural systems, is effectively actualized progressively intelligent condition. Creators of the NERO even recommend that this idea could be utilized later on for preparing individuals in advanced errands. Paper gave definite review of neuro-development applications in games, alongside the nitty-gritty examination of benefits and downsides accompanying this methodology.

Recently referenced Genetic Algorithms once in a while speak to a technique for decision in business games, as it is viewed as that this methodology is frequently excessively moderate, and requires such a large number of CPU assets. In any case, since the presence of the Cloak, Dagger, and DNA game (made by Don O' Brien), which executed GAs so as to create developing gaming agents, scholastics explored potential utilizations of GAs in game AI. Other than effectively depicted neuro-development, a few examinations were directed dependent on applying GAs to mainstream games, for example, Counter Strike or Quake III Arena. It is accepted by Lucas and Kendall that appropriately utilized developmental calculations could improve generally speaking playability of the game, suggesting in that way that potential business materialness could in the long run increment.

Blue Eyes Intelligence Engineering \& Sciences Publication

(C) Copyright: All rights reserved.

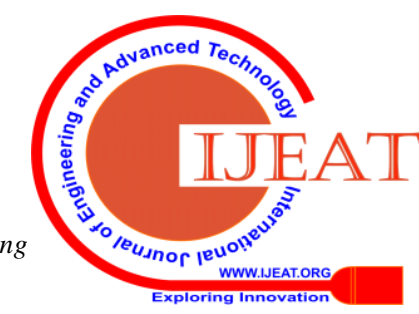




\section{Contribution of Artificial Intelligence and Virtual Worlds towards development of Super Intelligent AI Agents}

\section{A THEORETICAL FRAMEWORK FOR GAMING AgENTS - INTERACTION OF HUMAN-LEVEL INTELLIGENCE \& VIRTUAL WORLDS}

As it was seen, "Humans are humanity's favorite subject." This profound want to comprehend the embodiment of our reality and conduct, drove us to enormous accomplishments in various parts of science and workmanship. Number of scientific fields rotated around the need to comprehend and create human level limits.

Illustrative model is Robotics, where thought of making a completely practical humanoid robot has its underlying foundations grounded back in the history, sometime before the field itself was even settled [9]. With regards to the firmly related field of Artificial Intelligence, staggering outcomes were cultivated in various spaces during the most recent couple of decades. Purported "weak AI" gave various specific calculations and arrangements that are applied so as to improve various parts of innovation and human life in general. However, creating of human-level AI (or "strong AI", as it is regularly alluded) is as yet a fantasy, similar to it was on the very beginnings. A portion of the AI pioneers, for example, Marvin Minsky and Herbert Simon, were idealistic in the beginning of the field, predicting that human level AI will be accomplished until the finish of the twentieth century, which will in the end empower machines to do everything that human output. These expectations were not fulfilled, in that way opening various conversations that question why we despite everything cannot design human level machine insight, is human level knowledge fundamental, and toward the end is it even reachable. This is somewhat justifiable considering the way that not simply that we didn't accomplished the human-level AI, yet we are battling to arrive at the limits of life forms that we consider far more straightforward. In spite of the colossal mechanical headways we despite everything don't have a self-sufficient portable framework that has an adequacy and complexity of a "straightforward" cockroach.

PC games speak to one of the most illustrative examples of overcoming adversity of Artificial Intelligence frameworks which are practically identical with people. On the off chance that we investigate PC frameworks that can play board or games, momentous outcomes are practiced over the most recent couple of decades, considering impeccable data as well as defective data games also. At the point when IBM's Deep Blue framework [1], crushed Garry Kasparov in the epic chess fight rematch, open promotion considering the AI was at the pick. Number of different models can be recorded as well, for example, Checkers or prior referenced Backgammon and Go, where PC frameworks arrived at the degree of top-human execution [2]. Further, Alpha Zero calculation was accounted for to have exceptional outcomes playing Chess, Shogi, and Go. Despite the fact that prevalence of a portion of these frameworks did not depend exclusively on AI methods, look into in these games influenced the whole field of AI, firmly pushing new thoughts and approaches. Be that as it may, on the off chance that we accept Checkers for instance, notwithstanding the multifaceted nature of the game which is, in addition to other things, reflected in a reality that this game has about 500 billion of potential positions, this is as yet a

finite number of mixes [10]. Other than that, great tabletop games are for the most part flawless data, implying that all members of the game have understanding in everything that has occurred before they settle on a choice. Not at all like these finite, deterministic, constrained gaming spaces, people (just as other living creatures) live and settle on choices in a universe of uncertainness, with constrained data accessible, where infinite number of co-operations happens each day. Along these lines, so as to draw nearer to human-level knowledge we need in excess of a gaming board or a deck of cards. Regardless of how confounded and testing these recently referenced issues are, they speak to just one section of human knowledge. In their original work, Laird and Van Lent perceived intuitive 3D PC gaming universes as an ideal proving ground for research of the human-level AI. This view opens up an intriguing viewpoints with regards to various regions of AI inquire about [11].

To be specific, it is clear from the past segments of the paper, that virtual universes to be sure furnish us with likelihood to successfully explore various issues identified with keen specialists, and therefore various sections of human level AI issues. Simultaneously nature of systems on which the virtual universes are fabricated, could force an extreme impediments for full use of their potential regarding this matter. Further in the content a few parts of Laird's proposal will be broke down, along with potential ramifications. So as to more readily comprehend the capability of virtual universes on past issues, the subject of human-level AI from the point of view of chosen speculations must be briefly tended to first.

\section{A. Evolution, Embodiment Theory, and Situatedness}

Old style AI, additionally called GOFAI (Good Old-Fashioned Artificial Intelligence), indicated a great deal of weaknesses in seeking after of human-level AI. One of the principle reasons lies in the way that traditional AI speculations and master frameworks are profoundly grounded in data and image preparing. This methodology substantiated itself as an amazing and very efficient, thinking about various issues and applications. Be that as it may, it is regularly contested with regards to accomplishing of solid AI, as the idea of human insight lies on various foundations.

Determination that there is a chance, that we misjudged the very establishments of knowledge, was perceived by numerous researchers. To have a more profound comprehension about this, we should look for into the portion of the fundamental pieces of the human development. How did people become savvy? Numerous potential hypotheses and hence numerous theories are produced by the researchers in the pertinent fields. Advancement scholars attempted to reproduce our past, and to find key occasions and procedures that influenced improvement of human scholarly capacities, in that way conceding us from other known primates.

One could absolutely see that changes of physical qualities caused the adjustments in scholarly capabilities, and the other way around. Early speculations perceived bipedalism as a potential first change in development of people,

Published By:

Blue Eyes Intelligence Engineering \& Sciences Publication (C) Copyriaht: All rights reserved.

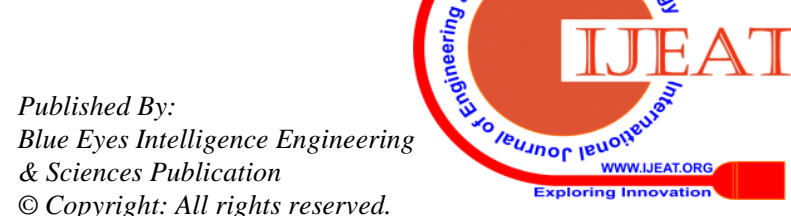


dating a bipedal strolling in the most punctual known primates. As an outcome of the embraced bipedalism, human body structure leaves from gorillas from numerous points of view. Remembering the way that bipedal strolling is one of the key qualities which are isolating people from different primates and that bipedalism is so strange for mammalians all in all, it is normal to scrutinize an explanation behind this sort of conduct. Number of various, and regularly inverse hypotheses was made - e.g., a few investigations detailed that bipedalism showed up as a vigorously efficient arrangement contrasting with quadrupedal motion, while others denied it. Be that as it may, final answer to this mind boggling issue is as yet staying unsolved. What is sure is the way that bipedalism went before the cerebrum development. In this way, one could infer that bipedalism vigorously influenced human behavior, and accordingly influenced the molding of our insight. Upstanding strolling changed the human point of view of the earth, and changed the manner in which people cooperate with it. Free forelimbs empowered numerous valuable exercises, for example, assembling and utilizing of instruments, and controlling the earth all in all, in that way definitively influencing the human advancement.

Considering only a short gander at a portion of the development foundations referenced in the past content, clearly human knowledge is indivisible from human body, and the other way around. As it was seen "biological mind is, first and foremost, an organ for controlling the biological body."

This sort of approach is reflected in Embodiment Theory, which showed up as a reaction to the traditional AI. A number of researchers think about epitome as an essential condition for creating of any kind of evident astute conduct, breaking down this issue from the human point of view as well as with illustrative models originating from various requests of creatures too. Concerning that, Pfefier and Scheier portrayed exemplification as: "A term used to refer to the fact that intelligence cannot merely exist in the form of an abstract algorithm but requires a physical instantiation, a body." obviously, this definition ought not be comprehended in a simplified sense, remembering the more profound significance with respect to the associations among neural and physical procedures.

The need to find an option in contrast to old style AI approaches was underlined in the spearheading exploration of Rodney Brooks. His work in the field of self-sufficient mechanical autonomy demanded physical establishing theory, rather than conventional image framework speculation. Streams completely investigated fundamental qualities of the two methodologies in his original work. Not at all like the conventional worldview where AI framework depends on an "arrangement of images" and its control, has physical establishing theory depended on the reason that portrayals of a savvy framework must be profoundly grounded in its physical encompassing. Streams expounded for what reason are genuinely grounded versatile robots better than image based robots, supporting his thoughts with number of created models [3]. Among other, situatedness and encapsulation are improving robot's versatility to the evolving condition, a trait so trademark for people [4]. It is additionally seen that other than its morphology, conduct of some substance is likewise influenced by nature in which it acts. As it was expounded, "Intelligence is dictated by the elements of connection with the world."

\section{B. Possible Implications to Virtual Worlds \& gaming agents}

On the off chance that we watch recently portrayed standards with regards to ebb and flow PC games innovation, we can without much of a stretch notification the examination potential behind these thoughts, yet in addition all the weaknesses which are compelling their full usage into the intelligent virtual universes [12]. To be specific, gaming agents show up as human-like symbols or a few animals, and act as indicated by their capacities inside the virtual condition, simultaneously influencing nature somehow or another. Anyway their epitome and situatedness are simplified. Gaming agents are "for all intents and purposes typified", or all the more exactly graphically encapsulated. They are not fabricated and along these lines are not acting in the way living creatures are. Further, the virtual universes themselves are centered on a visual likeness, and they come up short on a portion of the critical genuine attributes. This is a significant issue, since advancement of human insight is emphatically associated with perceiving and communicating with the dynamic 3-D world, its structures, and other living creatures. In the event that we need to follow the prior referenced standards of exemplification and situatedness in an all the more true way, at that point some adjustments of the virtual world mechanisms should be guaranteed. Gaming agents ought to most likely be furnished with a virtual dynamical encapsulation and all the more carefully set virtual situatedness, all inside intuitive virtual conditions modified to help such attributes. This ought not to be comprehended in as implied way as an insignificant presentation of some dynamical properties. All the more critically, gaming agents ought to be empowered (as much as it is conceivable) to detect their general surroundings and associate with different substances and the dynamical intuitive encompassing in a manner that looks like how living creatures act in reality [14]. All things considered, situatedness is perceived as one of the key prerequisites so as to define something as an operator, implying that it must be skilled to get contributions from sensors, and likewise somehow or another influence its condition. Concerning, alleged "tactile trustworthiness", speaks to one of the exceptionally significant issues, since it is infrequently actualized in virtual universes - gaming agents are generally worked to be omniscient, with no genuine comprehension of the world that is encompassing them.

Remembering the briefly expounded standards of subsumption engineering and epitome hypothesis, one ought to be cautious - applying of these standards changed in accordance with virtual universes ought not lead toward just simply receptive AI specialists, yet rather empowering them to coordinate and adventure diverse AI procedures and AI functionalities to a bigger degree. It ought to likewise be seen, that considering multifaceted nature of people and following
Published By:

Blue Eyes Intelligence Engineering \& Sciences Publication

(C) Copyright: All rights reserved.

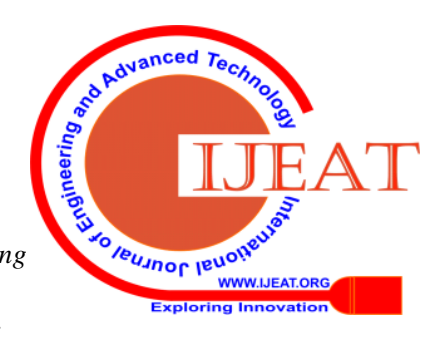




\section{Contribution of Artificial Intelligence and Virtual Worlds towards development of Super Intelligent AI Agents}

the thoughts explained in, recently referenced standards ought to be steadily applied by trying different things with artificial operators propelled with less complex living beings at first.

With regards to as of now referenced dynamical properties, intensive research concentrates on powerfully recreated graphical models were done before. Progressively reproduced characters as a choice to movement inscription and key-surrounding movement age strategies. In this examination, two virtual conditions were created and populated with gaming agents, mimicking bike hustling and knew about boats.

Liveliness of some picked human developments dependent on elements, are additionally altogether inquired about. One should see that dominant part of the investigations managing dynamical models in virtual situations, essentially meant to give increasingly reasonable graphical sensation. In this fundamental work, virtual marine was filled with virtual 3D fish models, giving in that way some unique bits of knowledge in the field of artificial life [13]. Recreated models were worked by simplified biomechanical and hydro-dynamical standards along with imitated sensors and genuine fish personal conduct standards.

\section{RESULT AND DISCUSSION}

Recently portrayed scholastic investigations gave significant experiences on progressively displayed liveliness. With regards to the business virtual universes, the vast majority of them utilize a type of material science motors established in the traditional mechanics hypothesis, regardless of whether they depend on inflexible body material science or mass total methodology. Illustrative models are Havok motor (utilized by Second life, Halo, Half-Life, and so forth.), or PhysX engine (used by Active Worlds, MafiaII,etc.). Notwithstanding the way that material science motors offer entire spectra of conceivable outcomes, virtual universes will in general be somewhat statics thinking about articles inside them, just as the idea of collaborations among players and gaming agents with nature. It is as yet risky to definitely demonstrate and mimic genuine properties. Other than clear unpredictability, bringing of some genuine properties through material science based models and progressed sensorial frameworks is additionally computationally costly. Presenting of truly complex items is seriously expanding number of interactions. Further, with the expanded number of re-enacted articles and associations, CPU assets are drastically running out. Consequently, it isn't astounding that the idea of the virtual universes and associations in them is as yet obliged, not displayed precisely enough, and not in the scale that is expected to completely apply standards behind the physical establishing theory.

Now, one should be cautious so as to keep away from conceivable misconception of a portion of the recently uncovered investigations. In particular, the objective isn't to repeat the world in the entirety of its assorted variety and intricacy (also this is difficult to do), yet rather to recognize and imitate a portion of its basic attributes, just as they can be copied. Human-level insight could be excessively reliable on different inward and outer elements to be imitated in that

manner. Be that as it may, following the similarity from humanoid mechanical technology - presenting of gaming agents to a portion of the fundamental true conditions and outfitting them with a portion of the basic systems and communication designs trademark for living creatures, could trigger transformative jump in their self-rule and knowledge. As it were, it could be one of the important "child" ventures toward creating of human-like insight and comprehension instruments, or it could in any event empower us to more readily comprehend establishments of human knowledge.

Other than recently portrayed angles, social behavior and along these lines social communication with other living creatures is likewise perceived as the key component of the cause of human insight. People are the main living creatures that are utilizing emblematic language, which among other empowered us to move our insight through ages. There will be no careful examination on these issues further in the content, as this point merits its very own study so as to be appropriately analyzed. However, in creator's view, a short conversation considering a few parts of the theme must be given in the accompanying lines. Specifically, it isn't astounding that operators, which can convey in a human like way, spoke to subject of broad research throughout the most recent couple of decades. Thusly, virtual universes filled in as an ideal proving ground for advancement of these prattle bots, as they are frequently called. One of the benefits is reflected in the way that virtual universes furnish gaming agents with enormous number of human clients to collaborate with. Another benefit originates from the way that different testing situations can be planned and tried in these virtual universes. Yearly Loebner Prize rivalry is composed focusing on PC controlled characters to breeze through the Turing assessment through literary correspondence. Since Turing test caused a variety of understandings, and a great deal of inverse feelings thinking about its legitimacy and efficiency [15]. Argumentation about its importance isn't in the focal point of this paper. In any case, what ought to be noted is that nonattendance of the epitome is perceived as one reason which are impairing gaming agents to breeze through the Turing assessment. Remembering that we utilize emblematic language to portray our general surroundings, the manner in which we sense it and its marvel, an after intelligent inquiry is forcing itself. Is it sensible to anticipate that any immaterial PC framework, which cannot collaborate and detect the world in a human like way, could be competent to perform completely human-level astute discussion with no stunts?

Toward the finish of this area, one should see that there is no extreme arrangement that ensures progress toward accomplishing of human-level AI specialists. So as to draw near to the human level insight, or possibly accomplish a few portions of it, various hypotheses, half and half arrangements, and strategies must be incorporated so as to completely misuse their qualities and simultaneously limit their shortcomings. Other than that, virtual universes themselves just as gaming agents acting in them ought to be deliberately structured all together for these techniques to be compelling.

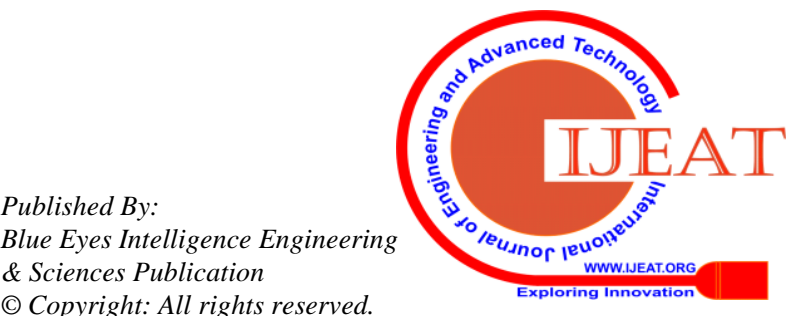

Blue Eyes Intelligence Engineering (c) Copyriaht: All rights reserved. 


\section{CONCLUSION}

This paper planned to give an extraordinary point of view regarding the matter of AI specialists in virtual universes. The main role was not to overcome any issues between AI scholastics and business based gaming industry, yet rather to assemble significant bits of knowledge originating from the two sides, basically assess them, interconnect them and point out the multidisciplinary lavishness and the examination capability of the explained problems. Therefore, creator is trusting that this exploration study will fill in as an important wellspring of data for a wide scope of specialists.

Extraordinary accentuation of the paper was on human-level AI investigated with regards to keen operators in virtual universes.

With regards to AI specialist issues that can be examined in virtual universes, number of potential applications is obliged distinctly by creative mind of research network, and ebb and flow specialized restrictions. Along these lines, it is imperative to make reference to, that usage of strategies and hypotheses introduced in this paper is frequently compelled with CPU assets. This is particularly with respect to a portion of the ongoing related issues, that operators frequently meet. Such specialized issues were perceived, yet not dissected in subtleties, as they are not in the principle focal point of the paper. All things considered, adhering to the Moore's law, these limitations are significantly decreasing during the years, and subsequently are not bargaining the hypothetical benefit of underlining research thoughts.

Prior it was referenced by Laird that at one point later on, PC games should advance, unavoidable focusing on cutting edge AI specialists with the need to try and match human-level knowledge so as to give next degree of practical experience for clients. On the off chance that one cautiously watches past segments of the paper, just as the necessary properties of artificial frameworks defined, in perfect situation those operators ought to be among other empowered with a few basic capacities: suitable thinking about its condition and their job in it, learning and shrewdly communicating with the dynamic condition incorporating an effectively adapting to vulnerabilities, and foreseeing the occasions and conduct of other unique elements in a powerful domain. Pragmatic justification of Laird's proposal is reflected in a few beneficial viewpoints to the further improvement of virtual universes. Human clients are increasingly drawn in while contending with different people, than with PC controlled adversaries that frequently carry on excessively unsurprising. Accordingly, a requirement for insightful specialists that can give progressively vivid and life-like virtual world experience appears to be somewhat self-evident. PC controlled AI rivals that can carry on in a human-like way are accounted for to be all the more testing and pleasant. Another viewpoint is identified with the way that virtual universes are getting progressively powerful and complex, with expanded populace of human clients and NPCs too. Hence, there is a requirement for self-sufficient operators that can adapt to unpredicted situations. Laird's expectations are bit by bit advancing, as human-level characters are drawing an expanded consideration. Prior referenced constant methodology game StarCraft, speaks to an illustrative model. As needs be, StarCraft AI rivalries are sorted out planning to make operators with the capacity to effectively play the game and contend with people and other scripted NPCs. As Samuel forcefully saw, 'Programming computer to play games is a stage in the understanding of the methods that must be employed for the machine simulation of intellectual behaviour." Bearing as a top priority the enormous prevalence of this game, it isn't astonishing that it is perceived as a reasonable proving ground condition. The capability of StarCraft as a stage for research of human like NPCs is perceived since the beginning of the game. In spite of the fact that there is far until virtual characters arrive at top human execution in this complex virtual world, StarCraft speak to an examination subject of high intrigue. Supporting this, it ought to be noticed that DeepMind and Blizzard inquire about groups are effectively taking a shot at the fortification learning condition created based on the StartCraftII. With further progressions in profound picking up, including human-level control, operators are drawing near to certain portions of human capacities. It ought to likewise be referenced that intellectual and social demonstrating pulled in a ton of consideration over the most recent couple of years. In spite of the fact that this intriguing, exceptionally multidisciplinary point was not a subject of examination in this paper, one should perceive intellectual models as a conceivably incredible strategy that can be utilized in a turn of events (particularly on a more elevated level) of future human-like specialists. Intellectual models got from accessible player's gaming information can empower investigation of different key properties recorded, for example, "adjustment to ecological imperatives" in that way expanding operator's self-governance. Number of specialists saw that not very many of scholastics legitimately assaulted the topic of general knowledge. Concerning, a few creators legitimately guarantee that human-level $\mathrm{AI}$ is inquired about in the PC games space with more exertion than in some other, particularly with general game playing. Research in the human level clever characters, can benefit the whole AI field. Thusly, this paper was managing critical part of Laird's fundamental work - the way that intelligent virtual universes could speak to an amazing proving ground for seeking after of human-level machine knowledge. All the more significantly, they are getting progressively mind boggling and dynamical, with continuous dynamic and other human qualities progressively required. Further, PC characters in these universes are presented to various associations with human clients, among themselves, and with their environmental factors. As author would see it, this makes virtual universes a fairly one of a kind proving ground for various fragments of AI investigate and their potential joining - e.g., cutting edge humanoid robots cannot be securely presented to such connections, and in such scale inside this present reality (particularly in regards to the collaboration with people and other living creatures). There are unique, frequently very inverse conclusions in regards to the chance of accomplishing human-level artificial insight. All things considered, examine in the human-level AI speaks to a gigantic undertaking. This is reflected in a reality that it isn't risky just to accomplish the entirety of the human fundamental capacities, yet additionally to appropriately incorporate them. Numerous scientists are sure that human-level AI will in the long run be accomplished,

Blue Eyes Intelligence Engineering \& Sciences Publication

(C) Copyright: All rights reserved.

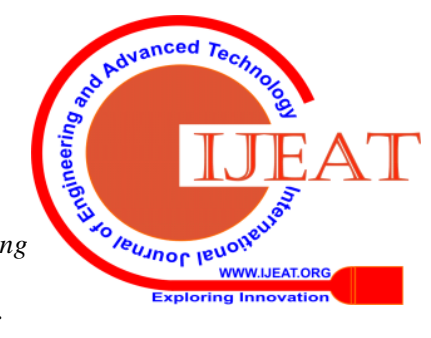




\section{Contribution of Artificial Intelligence and Virtual Worlds towards development of Super Intelligent AI Agents}

yet it requires for new ways to deal with be executed and incorporated along with the current ones. Regardless of whether it ought to be demonstrated later on, that this colossal undertaking is beyond the realm of imagination, one could be sure that examination in human-level AI isn't just helping us to all the more likely comprehend standards of human insight, but at the same time is delivering various "symptoms" across practically all scientific fields.

With respect to, the point of this paper was not to guarantee reachability of human-level AI, but instead to investigate boondocks and to underlain benefits and weaknesses of momentum best in class virtual universes and canny operators possessing them, with regards to human-level AI inquire about. Toward the end, creator is completely mindful that there is no investigation that could be ascribed as careful enough. With respect to, there are a few themes and speculations that are excluded and expounded in this work. It ought to be certain that there was no expectation to ignore or diminish significance of speculations that are not broke down in this exploration. Paper and its hypothetical substance are uncovered and composed in the way that as author would like to think best covers the underlining thoughts behind this examination study.

\section{REFERENCES}

1. M. Campbell, A. J. Hoane, Jr., and F.-H. Hsu, “Deep blue,' Artif. Intell., vol. 134, nos. 1-2, pp. 57-83, 2002.

2. J. Schaeffer et al., 'Checkers is solved,' Science, vol. 317 , no. 5844, pp. 1518-1522, 2007.

3. R. Pfeifer, F. Iida, and G. Gomez, "Designing intelligent robots-On the implications of embodiment,' Adv. Robot., vol. 24, no. 7, pp. 783-790, 2006.

4. R. Pfeifer, M. Lungarella, and F. Iida, "Self-organization, embodiment, and biologically inspired robotics,' Science, vol. 318 , no. 5853, pp. 1088-1093, 2007.

5. Y. LeCun, Y. Bengio, and G. Hinton, "Deep learning," Nature, vol. 521, pp. 436-444, May 2015.

6. V. Mnih et al., "Human-level control through deep reinforcement learning,'” Nature, vol. 518, pp. 529-533, 2015.

7. C. J. Bohil and F. A. Biocca, "Cognitive modeling of video game players,'’Dept.Telecommun.,Inf.Media,MichiganStateUniv.,EastLans ing, MI, USA, Tech. Rep., 2007.

8. L. A. Zadeh, "Toward human level machine intelligence-Is it achievable? The need for a paradigm shift,' IEEE Comput. Intell. Mag., vol. 3, no. 3, pp. 11-22, Aug. 2008.

9. M.L.Minsky, P.Singh, and A.Sloman, "The St. Thomas common sense symposium: Designing architectures for human-level intelligence,' AI Mag., vol. 25, no. 2, pp. 113-124, 2004.

10. Z. Kasap and N. Magnenat-Thalmann, "Intelligent virtual humans with autonomy and personality: State-of-the-art,' in New Advances in Virtual Humans: Artificial Intelligence Environment, N. Magnenat-Thalmann, L. C. Jain, N. Ichalkaranje, Eds. Springer-Verlag, 2008, ch. 2

11. J.Schaeffer, R.Lake, P.Lu, andM.Bryant, "CHINOOK:The world man machine checkers champion,'” AI Mag., vol. 17, no. 1, pp. 21-29, 1996.

12. J. E. Laird, "Research in human-level AI using computer games," Commun. ACM, vol. 45, no. 1, pp. 32-35, 2002.

13. P. Faloutsos, M. van de Panne, and D. Terzopoulos, "The virtual stuntman: Dynamic characters with a repertoire of autonomous motor skills,' Comput. Graph., vol. 25, no. 6, pp. 933-953, 2001.

14. R. Lopes and R. Bidarra, "Adaptivity challenges in games and simulations: A survey,'” IEEE Trans. Comput. Intell. AI in Games, vol. 3, no. 2, pp. 85-99, Jun. 2011.

15. R. M. French, "The turing test: The first 50 years," Trends Cogn. Sci., vol. 4, no. 3, pp. 115-122, 2000.

16. Prajval Mohan, Pranav Narayan, Lakshya Sharma, Tejas Jambhale, Simran Koul, "Iterative SARSA: The Modified SARSA Algorithm for Finding the Optimal Path". International Journal of Recent Technology and Engineering (IJRTE). ISSN: 2277-3878, Volume-8 Issue-6, March 2020.

Published By:

Blue Eyes Intelligence Engineering \& Sciences Publication (C) Copyriaht: All rights reserved.
Simran Koul was born in Jammu, India, on 10th November 1998. She completed her senior high school in FAIPS DPS, Ahmadi, Kuwait. She is currently pursuing for her Bachelor's Degree in Computer Science and Engineering at Vellore Institute of Technology, Vellore, India. She is currently working on projects which involve concepts of Robotics, Artificial Intelligence and Natural Language Processing.

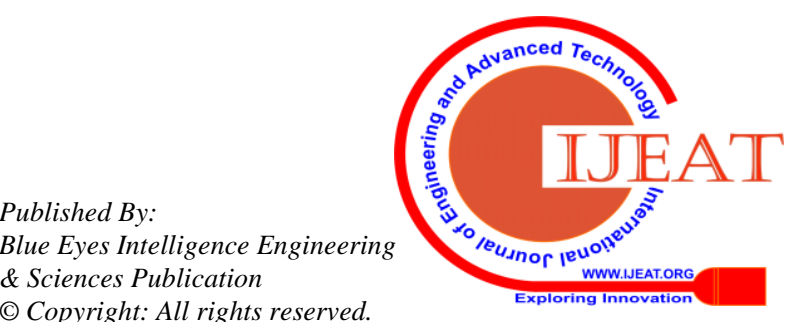

